\title{
First-line trifluridine/tipiracil plus bevacizumab for unresectable metastatic colorectal cancer: SOLSTICE study design
}

\author{
Thierry André*,1, Mark Saunders ${ }^{2}$, Akira Kanehisa ${ }^{3}$, Eric Gandossi ${ }^{3}$, Ronan Fougeray ${ }^{3}$, \\ Nadia Causse Amellal ${ }^{3}$ \& Alfredo Falcone ${ }^{4}$ \\ ${ }^{1}$ Sorbonne Université \& Saint-Antoine Hospital, 184 rue du Faubourg Saint-Antoine, 75012 Paris, France \\ ${ }^{2}$ Christie Hospital NHS Foundation Trust, Manchester, M20 4BX, UK \\ ${ }^{3}$ Institut de Recherches Internationales Servier, 50 rue Carnot, 92500 Suresnes, France \\ ${ }^{4}$ University Hospital of Pisa, Via Roma 67, 56126 Pisa, Italy \\ *Author for correspondence: thierry.andre@aphp.fr
}

Trifluridine/tipiracil (TT) is an orally administered combination of the thymidine-based nucleoside analogue trifluridine and the thymidine phosphorylase inhibitor tipiracil hydrochloride, which increases the bioavailability of cytotoxic trifluridine. Encouraging antitumor activity of first-line TT + bevacizumab (TTB) has been observed in a Phase II study in patients with unresectable metastatic colorectal cancer ineligible for combination oxaliplatin- or irinotecan-based therapy. Here, we describe the design of SOLSTICE (NCT03869892), an open-label, Phase III trial in unresectable metastatic colorectal cancer patients who are not candidates for, or do not require, intensive therapy. The 854 patients were randomized 1:1 to receive first-line TT-B versus capecitabine + bevacizumab. The primary objective is to demonstrate superior progression-free survival with TT-B over capecitabine + bevacizumab. The first patient was enrolled in March 2019.

Lay abstract: Trifluridine/tipiracil is an oral chemotherapy drug combination that acts by affecting the DNA of tumor cells. In a previous study, initial (first-line) treatment with trifluridine/tipiracil plus the tumor-starving (anti-angiogenic) drug bevacizumab was effective in patients with metastatic colorectal cancer that could not be surgically removed (i.e., was unresectable) and who could not receive intensive therapy. The SOLSTICE trial is designed to compare the efficacy and safety of first-line trifluridine/tipiracil + bevacizumab versus another treatment, capecitabine + bevacizumab, in patients with unresectable metastatic colorectal cancer who are not candidates for intensive therapy.

First draft submitted: 29 November 2019; Accepted for publication: 19 December 2019; Published online: 9 January 2020

Keywords: bevacizumab $\bullet$ capecitabine $\bullet$ first-line $\bullet$ metastatic colorectal cancer $\bullet$ trifluridine/tipiracil

Colorectal cancer (CRC) is the third most commonly diagnosed cancer and the second leading cause of cancerrelated death worldwide [1]. Approximately $20-30 \%$ of patients with CRC present with metastases at diagnosis, and despite surgery \pm adjuvant chemotherapy nearly $25 \%$ of patients presenting with stage I-III CRC will develop metastases, which contributes to the high mortality rates associated with the disease [2-4]. The 5-year survival rate for patients with localized disease is $90 \%$, decreasing to $71 \%$ and $14 \%$ for patients for patients with regional and distant metastases, respectively [5].

Selection of treatment for metastatic CRC ( $\mathrm{mCRC}$ ) is dependent on disease- and patient-related factors such as the disease stage at presentation, the extent of metastatic spread, performance status (PS) and the presence of comorbidities $[4,6]$. Whereas potentially curative surgery is the treatment of choice for patients with resectable disease, treatment goals for patients with unresectable mCRC are typically not curative but include tumor shrinkage (in patients with high tumor burden), disease control and prevention of tumor progression with prolongation of survival and maintenance of quality of life (QOL) [4].

Future Medicine 


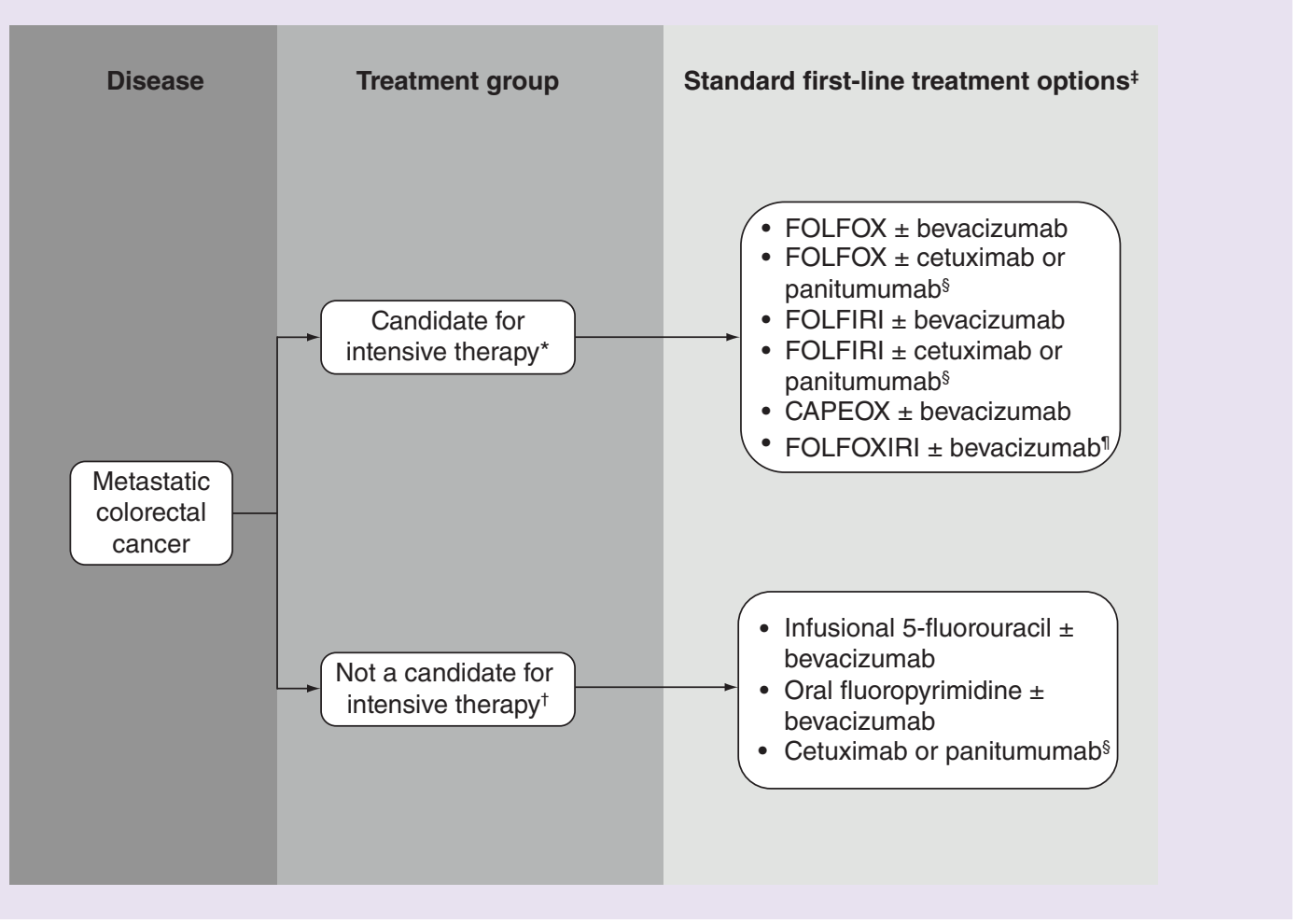

Figure 1. Standard first-line therapy options for patients with metastatic colorectal cancer. First-line options are based on European Society for Medical Oncology, National Comprehensive Cancer Network and Japanese Society for Cancer of the Colon and Rectum guidelines $[4,7,8]$.

*A patient with good tolerance for standard oxaliplatin- and/or irinotecan-containing chemotherapy in combination with targeted therapy for whom tumor shrinkage would be potentially beneficial.

†Includes patients with low tumor burden (intensive treatment not necessary for impending clinical threat), those for whom conversion to resectability is not possible, and those assessed as unfit for intensive therapy (i.e., frail with comorbidities) who may benefit from treatment other than best supportive care alone.

$\ddagger$ Chemotherapy may be administered alone, but unless contraindicated, combination therapy with targeted agents (i.e., bevacizumab, cetuximab, or panitumumab) is recommended.

§only patients with wild-type KRAS/NRAS/BRAF and left-sided tumors should be treated with cetuximab or panitumumab.

I Reserved for very fit and motivated patients where tumor shrinkage is the goal.

CAPEOX: Capecitabine + oxaliplatin; FOLFIRI: Infusional 5-fluorouracil + leucovirin + irinotecan; FOLFOX: Infusional 5-fluorouracil + leucovorin + oxaliplatin; FOLFOXIRI: Infusional 5-fluorouracil + leucovorin + irinotecan + oxaliplatin.

Patients with unresectable mCRC are divided into two groups and their treatment is selected according to whether or not they are candidates for an intensive treatment approach (Figure 1) $[4,7,8]$. Fluoropyrimidines are the cornerstone of treatment for $\mathrm{mCRC}$ [9], and standard first-line intensive therapy for patients with metastatic disease involves doublet or triplet cytotoxic chemotherapy regimens comprising a fluoropyrimidine plus oxaliplatin and/or irinotecan administered in combination with either a VEGF inhibitor (bevacizumab) or an anti-EGFR inhibitor (cetuximab or panitumumab) $[4,7,8]$. Combination therapy with a fluoropyrimidine compound and bevacizumab is the standard first-line treatment option for patients with unresectable mCRC who are not candidates for intensive chemotherapy but may benefit from treatment other than best supportive care alone $[4,7,8]$. Anti-EGFR therapy may be considered for patients with left-sided $R A S / B R A F$ wild-type tumors $[4,7,8]$.

Patients with $\mathrm{mCRC}$ who are not candidates for intensive therapy are often frail and/or elderly with comorbidities or with a low unresectable tumor burden and are therefore under-represented in clinical trials, which has led to a lack of innovative therapies for this population of patients [10-15]. There is therefore a clear unmet need for effective first-line treatment options for patients with mCRC who are not candidates for intensive therapy. 


\section{Trifluridine/tipiracil \& bevacizumab}

Trifluridine/tipiracil (FTD/TPI) is an oral formulation of the thymidine-based nucleoside analogue trifluridine (FTD) and the thymidine phosphorylase inhibitor tipiracil hydrochloride (TPI) at a molar ratio of 1:0.5 [16]. FTD is the active component of FTD/TPI, and by preventing rapid degradation of FTD to an inactive form by thymidine phosphorylase after oral administration, TPI improves systemic exposure to cytotoxic FTD [16]. The cytotoxic mechanism of action of FTD differs from that of the fluoropyrimidines, which act primarily as inhibitors of thymidylate synthase, the rate-limiting enzyme in the synthesis of DNA [16,17]. After phosphorylation by thymidine kinase, FTD is incorporated into DNA after further phosphorylation, resulting in DNA dysfunction [16]. FTD incorporation in DNA appears to be primarily responsible for the drug's antitumor activity $[16,18,19]$. On the basis of results from the global double-blind Phase III RECOURSE trial, which showed a significant overall survival (OS) benefit with FTD/TPI compared with placebo in heavily pretreated patients with mCRC [20], FTD/TPI monotherapy is approved in the EU, USA, Japan and other countries for the treatment of patients with mCRC refractory to standard therapies [21,22].

The VEGF-targeted agent bevacizumab inhibits angiogenesis and may normalize tumor vascular function, thereby improving tumor blood supply [23]. This may facilitate increased accumulation of FTD and its subsequent phosphorylation in tumors during combination therapy with FTD/TPI + bevacizumab (TT-B) [19,24]. Compared with FTD/TPI alone, the enhanced antitumor activity and increased phosphorylated FTD levels observed with TT-B in CRC xenograft models suggest that bevacizumab increases FTD accumulation in tumor cell DNA [19]. Increasing the concentration of FTD in tumors without increasing systemic exposure, as appears to occur during TT-B therapy [19,24], may optimize antitumor activity while minimizing toxicity.

The Phase I/II C-TASK-FORCE trial of TT-B in patients with unresectable mCRC refractory to standard therapies showed encouraging antitumor activity and manageable toxicity of the combination regimen [24]. The primary study end point of progression-free survival (PFS) at 16 weeks was $42.9 \%$ for the 21 patients in the primary analysis set [24], which exceeded a prespecified threshold based on a trial of FTD/TPI monotherapy for pretreated mCRC [25]. The median OS in C-TASK-FORCE was 11.4 months (95\% CI: 7.6-13.9) [24]. These promising results with TT-B in heavily pretreated mCRC patients led to the initiation of the randomized, openlabel, Phase II TASCO1 trial (NCT02743221) to evaluate the efficacy and safety of first-line therapy with TT-B or capecitabine + bevacizumab (C-B) in 154 patients with unresectable mCRC who were not eligible for intensive therapy [26]. In a preliminary analysis from this ongoing trial, median PFS was 9.2 months with TT-B and 7.8 months with C-B (hazard ratio [HR]: 0.71; 95\% CI: 0.48-1.06). Preliminary median OS was 18 months with TT-B, and 16.2 months with C-B (HR: 0.56; 95\% CI: 0.32-0.98). The overall response rate (ORR) was 33.8\% with TT-B and $30.3 \%$ with C-B, and the disease control rate (DCR) was 85.7 and $77.6 \%$, respectively. TT-B had a manageable safety profile, with more hematological toxicities and nausea and vomiting than $\mathrm{C}-\mathrm{B}$, but a lower rate of hand-foot syndrome [26].

Overall, the preliminary efficacy and safety findings of TT-B for the first-line treatment of $\mathrm{mCRC}$ in patients who are not candidates for intensive therapy observed in the TASCO1 trial are highly encouraging. A confirmatory Phase III trial is therefore warranted in this first-line setting.

\section{SOLSTICE trial}

We describe the design of the randomized, open-label, global Phase III SOLSTICE trial (NCT03869892), which will compare TT-B with C-B for the first-line treatment of patients with unresectable $\mathrm{mCRC}$ who are not candidates for intensive therapy.

\section{Objectives}

The primary objective of the SOLSTICE trial is to demonstrate the superiority of first-line therapy with TT-B over $\mathrm{C}-\mathrm{B}$ with regard to investigator-assessed PFS. The key secondary objective is to demonstrate superior OS with TT-B versus $\mathrm{C}-\mathrm{B}$. Other secondary objectives are to confirm the clinical benefit of treatment of $\mathrm{mCRC}$ with TT-B versus $\mathrm{C}-\mathrm{B}$ by evaluating ORR, and to compare the safety and the impact on QOL of the two treatment regimens. Additionally, the trial has an exploratory objective to compare the efficacy of TT-B and C-B according to $R A S$, $B R A F 600 E$ and mismatch repair status. 
Key eligibility criteria

All patients must be aged $\geq 18$ years, have histologically confirmed, previously untreated, unresectable mCRC and not be a candidate for standard full-dose combination chemotherapy with irinotecan or oxaliplatin according to the investigator's judgment. Reasons for noneligibility to these standard treatments could be, but are not limited to, age, PS, low tumor burden, comorbidities or nonclinical reasons. Patients who have received any previous systemic anticancer therapy for unresectable $\mathrm{mCRC}$ are not permitted, but previous adjuvant or neoadjuvant chemotherapy is permitted if the patient was disease-free for $\geq 6$ months after completion of chemotherapy. Patients must have $\geq 1$ measurable metastatic lesion, as defined by Response Evaluation Criteria in Solid Tumors (RECIST), and no symptomatic central nervous system metastases. Patients must also have an Eastern Cooperative Oncology Group (ECOG) PS score of $0-2$ and an estimated life expectancy of $\geq 12$ weeks. RAS mutation status must be known or determinable based on local biological assessment of tumor biopsy. Tumors will be assessed for the presence of wild-type KRAS (exon 2, 3 and 4) and NRAS (exon 2, 3 and 4) or KRAS and NRAS mutants (any exon and any mutation). Additionally, patients must have adequate renal (creatinine clearance $\geq 50 \mathrm{ml} / \mathrm{min}$ ), hepatic and hematologic function, and if other malignancies, in remission for $>5$ years. Excluded comorbidities are clinically significant cardiovascular disease (New York Heart Association class III or IV congestive heart failure, severe/unstable angina, stroke or myocardial infarction in the previous 6 months, uncontrolled diabetes mellitus, hypertension or arrhythmia); interstitial lung disease; severe or uncontrolled active infections; serious nonhealing wounds, ulcers or bone fractures; and deep vein thromboembolic event $\leq 4$ weeks before randomization. No major surgery is permitted $\leq 4$ weeks before randomization. Previous radiotherapy is permitted if completed $\geq 4$ weeks before randomization and $\geq 1$ measurable lesion is excluded from the field. Concurrent systemic immunosuppressive therapy is not permitted, although steroids administered in a prophylactic setting or at a chronic low dose are allowed.

\section{Study design}

SOLSTICE is a randomized, open-label, multicenter Phase III trial. Enrollment began in March 2019 and is ongoing. Patients are being recruited at 200 sites in 25 countries: Argentina, Australia, Austria, Brazil, Bulgaria, Czech Republic, Denmark, Estonia, France, Germany, Hungary, Ireland, Italy, Latvia, Lithuania, Poland, Portugal, Romania, Russia, Slovakia, Spain, Sweden, The Netherlands, Ukraine and the UK. Patients will be randomly allocated to treatment in a 1:1 ratio to receive TT-B or C-B, with randomization stratified by ECOG PS (0 vs 1 vs 2), tumor localization (right vs left) and reason for noneligibility for intensive therapy due to clinical condition (elderly, comorbidities, ECOG PS) or nonclinical condition (low tumor burden, patient's preference, other). Treatment with TT-B or C-B will continue until radiologic or clinical disease progression, unacceptable toxicity or other reasons for discontinuation (Figure 2).

The primary end point is PFS assessed by the investigator. To detect any bias in the investigator assessment of radiologic images, an additional Blind Independent Central Review (BICR) of PFS will be performed on a random sample of 300 patients. If the independent Data Monitoring Committee finds that there is inadequate concordance between BICR and investigator-assessed PFS, a BICR will be performed for all patients. The key secondary end point is OS. Other secondary end points include ORR and DCR, QOL assessments, safety and tumor biomarkers.

\section{Evaluations}

Antitumor response assessments via computed tomography or MRI are scheduled every 8 weeks after randomization until radiologic disease progression, death or initiation of a new anticancer treatment. Safety will be assessed throughout the study and adverse events graded based on National Cancer Institute Common Terminology Criteria for Adverse events v.4.03. QOL is assessed every 6 weeks after randomization using the European Organization for Research and Treatment of Cancer core QOL questionnaire and the 5-level EQ-5D questionnaire [27,28]. At baseline, patients will also undergo Comprehensive Geriatric Assessment (CGA) using the G8 questionnaire (patients $\geq 70$ years old) and Charlson Comorbidity Index [29,30].

\section{Statistical analysis methods}

As the sponsor of the SOLSTICE trial, IRIS (Institut de Recherche Internationale Servier) will be responsible for data collection and analysis. Intent-to-treat analysis of all efficacy end points will be performed for the full analysis set, which comprises all randomized patients, according to the treatment group to which they were randomized. The planned sample size of this trial $(\mathrm{n}=854)$ is driven by the primary end point of PFS, which is defined as the 


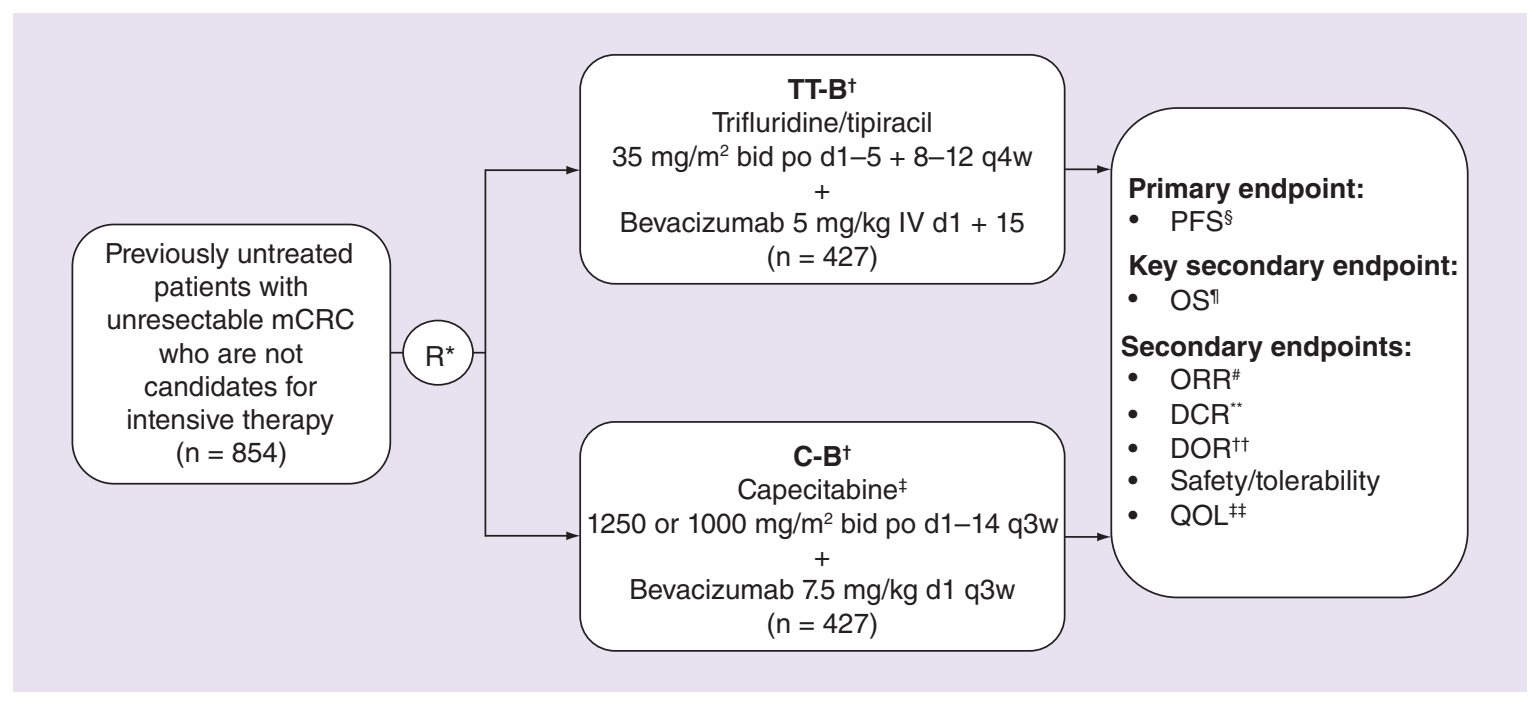

Figure 2. SOLSTICE trial design.

*Stratified by ECOG performance status ( 0 vs 1 vs 2), tumor localization (right vs left) and the reason why the patient is not a candidate for intensive therapy (clinical condition vs nonclinical condition).

†Treatment continued until radiologic or clinical disease progression, unacceptable toxicity, conversion to resectable $\mathrm{mCRC}$ or other reason for discontinuation.

¥The choice of the starting dose of capecitabine (either 1250 or $1000 \mathrm{mg} / \mathrm{m}^{2} \mathrm{bid}$ ) was at the investigators discretion.

$\S$ Time from randomization to the date of investigator-assessed radiologic tumor progression (according to RECIST 1.1 criteria) or death from any cause.

ITime from randomization to the date of death due to any cause.

\#The proportion of patients with objective evidence of CR or PR (according to RECIST 1.1 criteria) as assessed by the investigator.

**The proportion of patients with objective evidence of CR, PR or SD (according to RECIST 1.1 criteria) as assessed by the investigator.

${ }^{\dagger}$ The time from the first documentation of CR or PR to the first documentation of tumor progression as assessed by the investigator, or death due to any cause.

\#\#Assessed using EORTC QLQ-C30 and EQ-5D-5L questionnaires.

bid: Twice daily; C-B: Capecitabine + bevacizumab; d: Day; DCR: Disease control rate; CR: Complete response; DOR: Duration of response; ECOG: Eastern Cooperative Oncology Group; EORTC QLQ-C30: European Organization for Research and Treatment of Cancer core quality of life; EQ-5D-5L: 5-Level EQ-5D; IV: Intravenous; mCRC: Metastatic colorectal cancer; ORR: Overall response rate; OS: Overall survival; PFS: Progression-free survival; po: Oral; PR: Partial response; qxw: Every x weeks; QOL: Quality of life; R: Randomization; RECIST: Response Evaluation Criteria in Solid Tumor; SD: Stable disease; TT-B: Trifluridine/tipiracil + bevacizumab; TTF: Time to treatment failure.

time from randomization to the date of investigator-assessed radiologic tumor progression (according to RECIST 1.1 criteria) or death from any cause. For patients who were lost to follow-up without radiologic progression or reached the time point of analysis without a known record of death or radiologic progression, PFS will be censored at the date of last evaluable tumor assessment. A maximum of 628 PFS events will be required for the primary analysis to detect an HR of 0.77 with $90 \%$ power using a log-rank test and a 2-look group sequential design at one-sided cumulative $2.5 \%$ level of significance. The median duration of PFS is expected to be around 7.5 months with C-B and 9.7 months with TT-B. Based on the assumption that enrolment will continue for 18 months, and that about $10 \%$ of the subjects will have dropped out when reaching 36 months since the randomization, a total of 854 patients randomized in a 1:1 ratio will be needed to observe the 628th PFS events 10 months after the last patient randomization (follow-up duration for PFS analysis). To ensure $80 \%$ power to detect an HR of 0.79 in the OS analysis, OS will be tested 27 months after the randomization of the last patient (follow-up duration for OS analysis). The statistical methodology for the interim PFS analysis will be based on group sequential methodology with stopping boundaries defined by $\alpha$-spending function due to Lan Demets (1983) with O'Brien-Fleming type stopping boundary.

The primary hypothesis is that TT-B is superior to C-B in terms of PFS, and the key secondary hypothesis is that TT-B is superior to C-B in terms of OS. The primary and key secondary efficacy analyses will be the comparison of the distribution of PFS/OS between the two treatment groups using stratified log-rank tests at one-sided 2.5\% 
levels of significance. HRs for PFS and OS and corresponding 95\% CIs will be estimated using stratified Cox proportional hazard models, with strata based on the randomization stratification factors as obtained through an interactive web response system.

In order to maintain an $\alpha$-control of the $2.5 \%$ level of significance, OS will be tested in a hierarchical approach meaning that OS will be tested only if PFS is proven to be statistically significant. Other efficacy measures will be compared between treatment arms using Fisher's exact test and Clopper-Pearson CIs (ORR and DCR), or analyzed using Kaplan-Meier methodology and compared between treatment groups with a stratified log-rank test. All safety and QOL measures will be assessed using descriptive statistics by treatment arm.

\section{Ethical considerations}

SOLSTICE is being conducted in compliance with the trial protocol, and in accordance with the ethical principles of the Declaration of Helsinki, good clinical practice and applicable regulatory requirements. All patients enrolled in the trial are required to provide written informed consent.

\section{Discussion \& future perspectives}

There is currently no standard detailed definition of the population of patients with unresectable mCRC who are not candidates for intensive systemic therapy. This may be partly the result of a lack of rigorous criteria for the age cut-off defining 'elderly' patients, assessments to define 'frailty' and the definition of 'low' tumor burden. The choice of nonintensive therapy may be, in some cases, according to patient preference using a possible sequential approach (e.g., start with a single agent and bevacizumab, and move onto doublet \pm targeted therapy in the event of progression). International treatment guidelines refer to inappropriate candidates for intensive therapy in general terms as those who will be unable to tolerate standard-dose combination therapy with a fluoropyrimidine + oxaliplatin or irinotecan, those for whom intensive treatment is not necessary and where the goal is disease control, and those refusing intensive treatment for personal reasons $[4,7,8]$. Categorization of patients with unresectable $\mathrm{mCRC}$ as candidates for intensive therapy is therefore a subjective decision-making process that relies on the treating physician's evaluation of suitability for therapy [10,12]. This represents a major challenge when selecting the most appropriate treatment option for patients with unresectable mCRC.

Although CRC is predominantly a disease of older age (i.e., $\geq 65$ years) [5], the elderly patient population presents considerable heterogeneity with regard to comorbidity and functional status [13], such that a patient's biological age may not match their chronological age [31]. Moreover, the definition of elderly ( $\geq 65, \geq 70$ or $\geq 75$ years) differs in the published literature. Therefore, first-line treatment decisions for patients with unresectable mCRC should not be guided by age criteria alone, and should also take into account the patient's CGA results, PS, comorbidities, tumor characteristics, the extent of metastases and their own individual preferences $[12,31,32]$. In the SOLTSTICE study, the population of patients with low tumor burden mCRC may be defined as those with numerous small unresectable metastases, for example, CRC with numerous, but only, lung metastasis [33].

There is consensus among international treatment guidelines that combination therapy with a fluoropyrimidine plus bevacizumab is a first-line treatment option for patients with unresectable mCRC for whom more intensive treatment is not appropriate $[4,7,8]$. Although this recommendation is supported by data from randomized trials, eligibility criteria were often poorly defined in these studies in relation to suitability for intensive therapy. However, the studies included some degree of patient selection (e.g., conducted in an exclusively elderly population rather than the full range of patients who are not candidates for first-line intensive therapy), but the CGA/comorbidity assessment was generally not described $[11,13]$. Fluoropyrimidine monotherapy or reduced-dose fluoropyrimidine + oxaliplatin or irinotecan chemotherapy doublets may be used as first-line alternatives to fluoropyrimidine + bevacizumab therapy for mCRC patients assessed as unfit to receive intensive first-line chemotherapy $[4,7,13]$. Given the limited range of treatment options for these unfit patients, the optimal second-line approach is not clear and use of second-line treatment is uncommon, with patients tending to go on to receive best supportive care once they have progressed on first-line therapy $[4,7,13,34]$.

As well as providing confirmatory evidence of the efficacy and safety of first-line therapy with TT-B in a large international population of patients with unresectable mCRC assessed as being ineligible for intensive therapy, the SOLSTICE trial will collect exhaustive information on this patient population, including demographic data, CGA assessment data and data on tumor characteristics and poststudy anticancer therapies. Reasons for ineligibility for intensive chemotherapy as assessed by the SOLSTICE investigators may include, but are not limited to, age, PS, low tumor burden, comorbidities and nonclinical reasons, such as patient preference. The SOLSTICE trial population 
will therefore include a wide range of patients assessed as being inappropriate candidates for intensive therapy, and will help to provide insights into the appropriate selection of treatment for patient subgroups according to variables such as age ( $<70$ vs $\geq 70$ years), number of metastatic sites ( $1-2$ vs $\geq 3$ ), CGA results (G8 questionnaire score $<14$ vs $\geq 14$ in patients $\geq 70$ years old, Charlson comorbidity index score 0 vs $1-2)$, ECOG PS ( 0,1 vs 2$)$, location of primary disease (left vs right), BRAF/RAS (wild-type vs mutant) and MSI status (high vs low).

Ultimately, it is hoped that the results of the SOLSTICE trial will provide a much-needed new first-line treatment option for all patients with unresectable mCRC who are ineligible for intensive systemic therapy (ineligibility to combination therapy could be linked to the patient's condition, comorbidities, or a low tumor burden not requiring intensive chemotherapy). The SOLSTICE trial is the first opportunity to evaluate TT-B in the first-line treatment of unresectable CRC in a large Phase III clinical study.

Executive summary

- Treatment for patients with unresectable metastatic colorectal cancer ( $\mathrm{mCRC}$ ) is selected according to whether or not they are candidates for an intensive treatment approach.

- First-line intensive therapy for unresectable mCRC involves chemotherapy with a fluoropyrimidine + oxaliplatin and/or irinotecan administered in combination with targeted therapy (e.g., bevacizumab or cetuximab/panitumumab for wild-type RAS tumors).

- Combination therapy with a fluoropyrimidine + bevacizumab is the recommended first-line treatment for patients with unresectable $\mathrm{mCRC}$ who are not candidates for intensive chemotherapy.

- Patients with $\mathrm{mCRC}$ who are not candidates for intensive therapy are often frail and elderly, and under-represented in clinical trials, so their treatment choices are limited.

Trifluridine/tipiracil \& bevacizumab

- Trifluridine/tipiracil (FTD/TPI) is an oral formulation of the thymidine-based nucleoside analogue trifluridine and the thymidine phosphorylase inhibitor tipiracil hydrochloride, which increases the bioavailability of cytotoxic FTD.

- FTD/TPI is expected to be active in patients refractory to fluoropyrimidine-based therapy as the cytotoxic mechanism of action of FTD differs from that of fluoropyrimidines

- FTD/TPI monotherapy is approved in the EU, USA and Japan for the treatment of patients with mCRC refractory to standard therapies.

- In preclinical studies, bevacizumab enhanced the antitumor activity of FTD/TPI in human CRC xenografts.

- In the Phase II TASCO1 trial, patients with unresectable MCRC who were not eligible for intensive therapy $(n=154)$ were randomized to treatment with FTD/TPI + bevacizumab (TT-B) or capecitabine + bevacizumab (C-B). In preliminary TASCO1 results, TT-B had a manageable safety profile, and median progression-free survival was 9.2 months with TT-B versus 7.8 months with C-B (hazard ratio: $0.71 ; 95 \%$ confidence interval: $0.48-1.06$ ).

SOLSTICE trial

- SOLSTICE is a randomized, open-label, global, Phase III trial.

- Eligible patients should have previously untreated, unresectable mCRC and should not be candidates for standard full-dose oxaliplatin- or irinotecan-containing combination chemotherapy (ineligibility to combination therapy could be linked to the patient's condition, comorbidities or a low tumor burden not requiring intensive chemotherapy).

- Patients (planned $\mathrm{n}=854$ ) will be randomized 1:1 to receive TT-B or C-B until disease progression or unacceptable toxicity.

- The primary objective of the study is to demonstrate superiority of TT-B versus C-B with regard to progression-free survival (primary end point).

- The study will potentially confirm TT-B as a much-needed new first-line treatment option for patients with unresectable $\mathrm{MCRC}$ who are not candidates for intensive therapy.

\section{Author contributions}

All authors were involved in the conception and design of the study, the development of the methodology and writing, review and/or revision of the manuscript. All authors are involved in data acquisition and supervision during the ongoing SOLSTICE Study. A Kanehisa, E Gandossi, R Fougeray and NC Amellal are also responsible for the administrative and technical aspects of the study.

Financial \& competing interest disclosure

This study is sponsored by Servier. T André receives honoraria and/or consulting or advisory role from Amgen, Bristol-Myers Squibb, Clovis, HalioDX, MSD Oncology, Roche, Servier, Sanofi, Tesaro, Pierre Fabre, and Vantana; travel, accommodations, and expenses from Roche, Ventana, MSD oncology, Amgen and Bristol-Myers Squibb. M Saunders has attended advisory boards and chaired meetings for Roche, Merck, Servier, Amgen, Sanofi and Eisai. A Falcone has received honoraria from, and/or held a consulting 
or advisory role for Bayer, Bristol-Myers Squibb, Lilly, Merck, Pierre-Fabre, Roche, and Servier; his institution has received research funding from Astra-Zeneca, Bayer, Bristol, Lilly, Merck, MSD, Novartis, Roche, Sanofi, and Servier; and A Falcone has a leadership role in the following research groups/foundations: Fondazione GONO (Italy), Fondazione ARCO (Italy). A Kanehisa, E Gandossi, R Fougeray and NC Amellal are employees of Servier. The authors have no other relevant affiliations or financial involvement with any organization or entity with a financial interest in or financial conflict with the subject matter or materials discussed in the manuscript apart from those disclosed.

Medical writing support was provided by J Dalton, Springer Healthcare Communications and was funded by Servier.

\section{Open access}

This work is licensed under the Attribution-NonCommercial-NoDerivatives 4.0 Unported License. To view a copy of this license, visit http://creativecommons.org/licenses/by-nc-nd/4.0/

\section{References}

Papers of special note have been highlighted as: • of interest; $\bullet \bullet$ of considerable interest

1. Bray F, Ferlay J, Soerjomataram I et al. Global cancer statistics 2018: GLOBOCAN estimates of incidence and mortality worldwide for 36 cancers in 185 countries. CA Cancer J. Clin. 68(6), 394-424 (2018).

2. Bartlett DL, Chu E. Can metastatic colorectal cancer be cured? Oncology 26(3), 266-75 (2012).

3. Valderrama-Trevino AI, Barrera-Mera B, Ceballos-Villalva JC, Montalvo-Jave EE. Hepatic metastasis from colorectal cancer. Euroasian J. Hepatogastroenterol. 7(2), 166-175 (2017).

4. Van Cutsem E, Cervantes A, Adam R et al. ESMO consensus guidelines for the management of patients with metastatic colorectal cancer. Ann. Oncol. 27(8), 1386-422 (2016).

- European guidelines, which recommend treatment with a fluoropyrimidine + bevacizumab for patients with unresectable metastatic colorectal cancer (mCRC) for whom more intensive oxaliplatin-or irinotecan-containing therapy is inappropriate.

5. Siegel RL, Miller KD, Fedewa SA et al. Colorectal cancer statistics, 2017. CA Cancer J. Clin. 67(3), 177-193 (2017).

6. Stein A, Bokemeyer C. How to select the optimal treatment for first line metastatic colorectal cancer. World J. Gastroenterol. 20(4), 899-907 (2014).

7. National Comprehensive Cancer Network. NCCN clinical practice guidelines in oncology (NCCN Guidelines ${ }^{\circledR}$ ). Colon cancer (2019). www.nccn.org/professionals/physician_gls/def ault.aspx

8. Hashiguchi Y, Muro K, Saito Y et al. Japanese Society for Cancer of the Colon and Rectum (JSCCR) guidelines 2019 for the treatment of colorectal cancer. Int. J. Clin. Oncol. (2019).

- Japanese guidelines, which recommend treatment with a fluoropyrimidine + bevacizumab for patients with unresectable mCRC for whom more intensive oxaliplatin- or irinotecan-containing therapy is inappropriate.

9. Fakih MG. Metastatic colorectal cancer: current state and future directions. J. Clin. Oncol. 33(16), 1809-1824 (2015).

10. Bruera G, Russo A, Galvano A, Rizzo S, Ricevuto E. Clinical parameters to guide decision-making in elderly metastatic colorectal cancer patients treated with intensive cytotoxic and anti-angiogenic therapy. Oncotarget 8(23), 37875-37883 (2017).

11. Cunningham D, Lang I, Marcuello E et al. Bevacizumab plus capecitabine versus capecitabine alone in elderly patients with previously untreated metastatic colorectal cancer (AVEX): an open-label, randomised Phase 3 trial. Lancet Oncol. 14(11), 1077-1085 (2013).

- A Phase III trial showing that first-line capecitabine + bevacizumab (C-B) is an effective and well-tolerated therapy in elderly patients ( $\geq 70$ years) with unresectable $\mathrm{mCRC}$ who are not candidates for intensive therapy, which supported the rationale for the C-B comparator arms in the Phase II TASCO1 and Phase III SOLSTICE studies in mCRC patients who are unsuitable for intensive therapy.

12. Dagher M, Sabido M, Zollner Y. Effect of age on the effectiveness of the first-line standard of care treatment in patients with metastatic colorectal cancer: systematic review of observational studies. J. Cancer Res. Clin. Oncol. 145(8), 2105-2114 (2019).

13. Pinto C, Antonuzzo L, Porcu L et al. Efficacy and safety of bevacizumab combined with fluoropyrimidine monotherapy for unfit or older patients with metastatic colorectal cancer: a systematic review and meta-analysis. Clin. Colorectal Cancer 16(2), e61-e72 (2017).

- A meta-analysis showing that first-line therapy with a fluropyrimidine + bevacizumab is a feasible treatment option for elderly or unfit patients with mCRC, which supported the rationale for the C-B comparator arms in the Phase II TASCO1 and Phase III SOLSTICE studies in mCRC patients who are unsuitable for intensive therapy.

14. Tapia Rico G, Price T, Tebbutt N et al. Right or left primary site of colorectal cancer: outcomes from the molecular analysis of the AGITG MAX trial. Clin. Colorectal Cancer 18(2), 141-148 (2019).

15. Tebbutt NC, Wilson K, Gebski VJ et al. Capecitabine, bevacizumab, and mitomycin in first-line treatment of metastatic colorectal cancer: results of the Australasian Gastrointestinal Trials Group Randomized Phase III MAX Study. J. Clin. Oncol. 28(19), 3191-3198 (2010). 
16. Lenz HJ, Stintzing S, Loupakis F. TAS-102, a novel antitumor agent: a review of the mechanism of action. Cancer Treat. Rev. 41(9), 777-783 (2015).

17. Sobrero A, Guglielmi A, Grossi F, Puglisi F, Aschele C. Mechanism of action of fluoropyrimidines: relevance to the new developments in colorectal cancer chemotherapy. Semin. Oncol. 27(5, Suppl. 10), 72-77 (2000).

18. Miyamoto Y, Lenz HJ, Baba H. A novel antimetabolite: TAS-102 for metastatic colorectal cancer. Expert Rev. Clin. Pharmacol. 9(3), 355-365 (2016).

19. Tsukihara H, Nakagawa F, Sakamoto K et al. Efficacy of combination chemotherapy using a novel oral chemotherapeutic agent, TAS-102, together with bevacizumab, cetuximab, or panitumumab on human colorectal cancer xenografts. Oncol. Rep. 33(5), 2135-2142 (2015).

20. Mayer RJ, Van Cutsem E, Falcone A et al. Randomized trial of TAS-102 for refractory metastatic colorectal cancer. N. Engl. J. Med. 372(20), 1909-1919 (2015).

21. Shitara K, Doi T, Dvorkin M et al. Trifluridine/tipiracil versus placebo in patients with heavily pretreated metastatic gastric cancer (TAGS): a randomised, double-blind, placebo-controlled, Phase 3 trial. Lancet Oncol. 19(11), 1437-1448 (2018).

22. Falcone A, Ohtsu A, Van Cutsem E et al. Integrated safety summary for trifluridine/tipiracil (TAS-102). Anticancer Drugs 29(1), 89-96 (2018).

23. Jain RK. Normalizing tumor vasculature with anti-angiogenic therapy: a new paradigm for combination therapy. Nat. Med. 7(9), 987-989 (2001).

24. Kuboki Y, Nishina T, Shinozaki E et al. TAS-102 plus bevacizumab for patients with metastatic colorectal cancer refractory to standard therapies (C-TASK FORCE): an investigator-initiated, open-label, single-arm, multicentre, Phase 1/2 study. Lancet Oncol. 18(9), 1172-1181 (2017).

- A single-arm Phase I/II study showing that trifluridine/tipiracil + bevacizumab has promising antitumor activity with manageable toxicity in pretreated patients with unresectable mCRC, which supported the rationale for the Phase II TASCO1 trial of first-line trifluridine/tipiracil + bevacizumab or C-B in patients not eligible for intensive therapy.

25. Yoshino T, Mizunuma N, Yamazaki K et al. TAS-102 monotherapy for pretreated metastatic colorectal cancer: a double-blind, randomised, placebo-controlled Phase 2 trial. Lancet Oncol. 13(10), 993-1001 (2012).

26. Lesniewski-Kmak K, Moiseenko V, Saunders M et al. Phase II study evaluating trifluridine/tipiracil + bevacizumab and capecitabine + bevacizumab in first-line unresectable metastatic colorectal cancer $(\mathrm{mCRC})$ patients who are non-eligible for intensive therapy (TASCO1): results of the primary analysis [abstract O-022]. Ann. Oncol. 29(Suppl. 5), mdy149.021 (2018).

-. A randomized Phase II study showing that trifluridine/tipiracil + bevacizumab has promising antitumor activity and manageable toxicity in patients with unresectable mCRC who are not candidates for intensive therapy, which supported the rationale for the Phase III SOLSTICE trial in this population.

27. Aaronson NK, Ahmedzai S, Bergman B et al. The European Organization for Research and Treatment of Cancer QLQ-C30: a quality-of-life instrument for use in international clinical trials in oncology. J. Natl Cancer Inst. 85(5), 365-76 (1993).

28. Herdman M, Gudex C, Lloyd A et al. Development and preliminary testing of the new five-level version of EQ-5D (EQ-5D-5L). Qual. Life Res. 20(10), 1727-1736 (2011).

29. Bellera CA, Rainfray M, Mathoulin-Pelissier $S$ et al. Screening older cancer patients: first evaluation of the G-8 geriatric screening tool. Ann. Oncol. 23(8), 2166-2172 (2012).

30. Charlson ME, Pompei P, Ales KL, MacKenzie CR. A new method of classifying prognostic comorbidity in longitudinal studies: development and validation. J. Chronic Dis. 40(5), 373-383 (1987).

31. Marosi C, Koller M. Challenge of cancer in the elderly. ESMO Open 1(3), e000020 (2016).

32. Winther SB, Jorgensen TL, Pfeiffer P, Qvortrup C. Can we predict toxicity and efficacy in older patients with cancer? Older patients with colorectal cancer as an example. ESMO Open 1(3), e000021 (2016).

33. Henriques J, Vernerey D, de Gramont A et al. Prognosis of lung metastases in patients with metastatic colorectal cancer: an ARCAD meta analysis. Ann. Oncol. 27(Suppl. 2), ii122-ii122 (2016).

34. Shibutani M, Maeda K, Nagahara $\mathrm{H}$ et al. Two cases of long-term control of metastatic colorectal cancer via FTD/TPI plus bevacizumab in elderly patients. Case Rep. Oncol. 11(3), 800-805 (2018). 
